\title{
Noninvasive magnetic resonance imaging stratifies injury severity in a rodent model of male juvenile traumatic brain injury
}

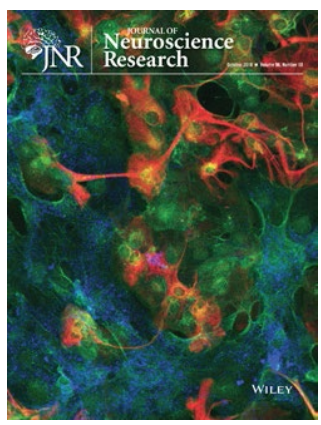

\author{
Jerome Badaut $^{1,2}$ (D) | Arash Adami $^{2}$ (D) | Lei Huang ${ }^{2,4}$ | André Obenaus ${ }^{2,3,5}$ (D)
}

\begin{abstract}
${ }^{1}$ CNRS UMR5287, University of Bordeaux, Bordeaux, France

${ }^{2}$ Department of Basic Sciences, Loma Linda University School of Medicine, Loma Linda, California

${ }^{3}$ Division of Biomedical Sciences, Center for Glial-Neuronal Interactions, UC Riverside, Riverside, California

${ }^{4}$ Department of Neurosurgery, Loma Linda University School of Medicine, Loma Linda, California

${ }^{5}$ Department of Pediatrics, University of California Irvine, Irvine, California
\end{abstract}

\section{Correspondence}

André Obenaus, Department of Pediatrics, University of California Irvine, Irvine, CA 92697.

Email: obenausa@uci.edu

Funding information

This study was supported by National Institute of Child Health and Human Development (NICHD) R01HD061946 (JB) and EraNet Neuron consortiums CNSA flame and TRAINS (JB), TRAIL- Laboratory of ExcellenceTRAIL (ANR-10-LABX-57 to JB)

\begin{abstract}
Age and severity are significant predictors of traumatic brain injury (TBI) outcomes in the immature brain. TBI studies have segregated TBI injury into three severity groups: mild, moderate, and severe. While mild TBI is most frequent form in children and adults, there is debate over the indicators used to denote mild injury. Clinically, magnetic resonance imaging (MRI) and computed tomography (CT) are used to diagnose the TBI severity when medically warranted. Herein, we induced mild, moderate, and severe $\mathrm{TBI}$ in juvenile rats ( $\mathrm{j} \mathrm{TBI}$ ) using the controlled cortical impact model. We characterized the temporal and spatial injury after graded $\mathrm{jTBI}$ in vivo using high-field MRI at 0.25 (6 hr), 1 and 3 days post-injury (dpi) with comparative histology. Susceptibilityweighted imaging (SWI) for blood and T2-weighted imaging (T2WI) for edema were quantified over the 0.25-3 dpi. Edema volumes increased linearly with severity at 0.25 dpi that slowly continued to decrease over the $3 \mathrm{dpi}$. In contrast, blood volumes did not decrease over time. Mild TBI had the least amount of blood visible on SWI. Fluoro-jade B (FJB) staining for cell death confirmed increased cellular death with increasing severity and increased FJB + cells in the corpus callosum (CC). Interestingly, the strongest correlation was observed for cell death and the presence of extravascular blood. A clear understanding of acute brain injury (jTBI) and how blood/edema contribute to mild, moderate, and severe jTBI is needed prior to embarking on therapeutic interventions. Noninvasive imaging should be used in mild jTBI to verify lack of overt injury.

KEYWORDS

blood, cell death, edema, hemorrhage, rat, white matter
\end{abstract}

\section{1 | INTRODUCTION}

Traumatic brain injury (TBI) is one of the most common neurological disorders among all age groups (Gotschall et al., 1995; Semple, Blomgren, Gimlin, Ferriero, \& Noble-Haeusslein, 2013) and is a consequence of an external mechanical force due to various origins, brain locations, severity leads to a heterogeneous disorder. The pediatric population is at high risk for TBI resulting in a leading cause of death and disability, especially with infants ( $<2$ years) and adolescents (Dewan, Mummareddy, Wellons, \& Bonfield, 2016; Thurman, 2016). These estimates are likely to be underreported to health services due in part to the mild nature of the head trauma in many of these cases. The most common causes of juvenile TBI (jTBI) are falls, sports, and motor vehicle accidents (Mannix, O'Brien, \& Meehan, 2013; Yue et al., 2016). Regardless of the severity of TBI, the initial brain injury in children can evolve into a lifelong disability and reduce 


\section{Significance}

The immature brain, relative to the adult is more susceptible of the effects of TBI. Trauma severity, clinically and in preclinical models, requires clear delineation blood and edema. MRI can map, both visually and quantitatively, injury volumes to assist clinical diagnosis and guide future therapeutic interventions.

quality of life of many patients (Babikian, Merkley, Savage, Giza, \& Levin, 2015). The pediatric and juvenile brain in TBI patients are at higher risk to increased brain swelling and edema formation than adults with the same severity injury (Bauer \& Fritz, 2004). Clinically, injury severity is often assessed using the Glasgow Coma Scale to categorize mild, moderate, or severe injuries. Neuroimaging can be used to assist in moderate to severe injury severity discrimination.

The evolution of TBI pathophysiology is dependent of the severity of the primary injury, which modulates behavioral outcomes in patients and in preclinical models (DeKosky \& Asken, 2017; Di Battista, Soo, Catroppa, \& Anderson, 2012; Siebold, Obenaus, \& Goyal, 2018). One aspect that complicates neurological and behavioral assessments is that TBI patients often present with a complexity of lesions of varying severity and regional distribution (Haghbayan et al., 2016; Isokuortti et al., 2018). Neuroimaging, particularly computed tomography (CT) is increasingly utilized in the emergency room for assessment of injury, but focuses mostly on the presence of extravascular blood. However, multimodal magnetic resonance imaging (MRI) can provide additional diagnostic information, including edema, hemorrhage, and assessment of white matter integrity. More importantly, the progression of the injury can be readily monitored temporally using MRI in animal models of TBI (Rodriguez-Grande et al., 2018; Wendel et al., 2018), albeit less frequently in young patients (Levin et al., 2008). In preclinical TBI models, positive correlations between $\mathrm{T} 2$ relaxation values, poor histological and behavioral outcomes have been reported (Kharatishvili, Sierra, Immonen, Grohn, \& Pitkanen, 2009).

Susceptibility-weighted imaging (SWI) is acutely sensitive to the presence of extravascular blood (Haacke, Xu, Cheng, \& Reichenbach, 2004). In fact, jTBI patients with moderate and severe injuries have reported intracranial hemorrhagic injuries (McKee \& Daneshvar, 2015). Dependent upon brain injury severity, edema is typically observed $24-48 \mathrm{hr}$ following injury and resolves by approximately 4-7 days, while hemorrhagic lesions can occur within the first 3-4 days and resolution can take months (Kurland, Hong, Aarabi, Gerzanich, \& Simard, 2012; Obenaus et al., 2007; Oehmichen, Walter, Meissner, \& Friedrich, 2003). SWI has been used in infants and children with TBI with heightened sensitivity for extravascular blood detection (Liu, Li, Tong, Yeom, \& Kuzminski, 2015; Tong et al., 2008). However, given the lack of progress in clinical trials to treat TBI, new approaches to guide model development are warranted to achieve success in translation from bench to bedside.
There are a limited number of developmental TBI models from postnatal day 17 (jTBI) to 35 (adolescent-TBI) with the most commonly used being either controlled cortical impact (CCI) or fluid percussion injury (FPI). $\mathrm{CCl}$ and $\mathrm{FPI}$ have been extensively used due to motor and cognitive impairments resembling many of the behavioral deficits observed in TBI patients (Yu et al., 2009) as well as reproducing contusion (CCI) and diffuse (FPI) TBI. Many studies have used the $\mathrm{CCl}$ model due to its ease of use, reproducibility, and the ability to easily adjust the degree of injury severity (Obenaus et al. 2012a). In the CCl model, the injury to the brain initially presents as necrotic cell death in the underlying tissue and white matter axonal injury, both reminiscent of the clinical TBI pathology. It is followed by apoptotic cell death in surrounding tissues due to multiple secondary events such as edema, ischemia, excitotoxicity, and altered gene expression in the developing brain (Dikranian et al., 2008; Sandhir, Onyszchuk, \& Berman, 2008) and in adult mice (Igarashi, Potts, \& Noble-Haeusslein, 2007; Sandhir et al., 2008; Yu et al., 2009). Despite the abundance of $\mathrm{CCl}$ studies, only a few studies have characterized a range of trauma severity, primarily focusing on histological evaluation in adult rodents (Igarashi et al., 2007; Siebold et al., 2018; Yu et al., 2009). Virtually, all published studies have focused on adult TBI and what remains to be elucidated are the effects of graded injuries to the juvenile brain, especially with the increased interest to mild TBI in preclinical research.

The objective of this study was to use clinically available MR imaging methods to fully characterize the acute temporal evolution and lesion composition (blood, edema) in a postnatal day 17 (P17) juvenile model of TBI. We are the first to report that acute MRI of jTBI using $\mathrm{T} 2 \mathrm{WI}$ and SWI are strong reporters of injury severity where the relative composition of blood/edema are similar at $1 \mathrm{dpi}$ independent of the final lesion size. Moreover, blood volumes at $6 \mathrm{hr}$ after injury were prognostic for injury severity. To our knowledge, this is the first study to explicitly examine injury severity in a juvenile model of TBI.

\section{2 | METHODS AND EXPERIMENTAL DESIGN}

Experiments and care of animals were conducted according to the principles and procedures of the Guidelines for Care and Use of Experimental Animals and approved by Loma Linda University Anima Care Committee. Sprague Dawley male rat pups at postnatal day 17 (P17) $(n=24)$ were housed in a temperature-controlled $\left(64-69^{\circ} \mathrm{F}\right)$ animal facility on a 12-hr light/dark cycle. In the current work, we focused on males because it represents clinically most of the cases of TBI in pediatrics (L. Wilson et al., 2017).

\section{1 | Rat model of graded juvenile TBI}

Rat pups were anesthetized with isoflurane (3\% induction, $2 \%$ maintenance) and placed in a stereotaxic apparatus (David Kopf Instrument, Tujunga, USA). Following a midline incision over the 
skull, a 3-mm diameter hole was drilled over the right hemisphere centered at $1 \mathrm{~mm}$ posterior from bregma and $2 \mathrm{~mm}$ lateral to midline (-1 mm AP, $-2 \mathrm{~mm} \mathrm{ML)} \mathrm{using} \mathrm{a} \mathrm{handheld} \mathrm{drill} \mathrm{(Fine} \mathrm{Science} \mathrm{Tools} \mathrm{Inc.,}$ Foster City, CA). The dura was left intact and any overt bleeding was lightly rinsed with isotonic saline. $\mathrm{CCl}$ injuries were induced using a pneumatic impactor with a 2-mm blunt tip angled at 20 degrees to the surface of the brain, as described previously (Obenaus et al., 2007). The impactor compressed the brain rapidly at $2.37 \mathrm{~m} / \mathrm{sec}$ with a dwell time of $250 \mathrm{~ms}$. The injury severity grades were differentiated by changing the depth of compression: mild: 1.0 , moderate: 1.5 , and severe: $2.0 \mathrm{~mm}$ from the cortical surface. Injury severity measures were derived from work in our own laboratory in pediatric (Ajao et al., 2012) and adult rats (Donovan et al., 2012; Obenaus et al., 2007) as well as adult mice (Bajwa, Lee, Halavi, Hartman, \& Obenaus, 2019). A recent review of the mouse TBI literature recommended similar depth settings as those we used in the current study (Siebold et al., 2018). Immediately after impact, the wound was sutured with nonabsorbable 5-0 silk sutures (Covidien, Mansfield, MA) and the pups then placed on a warm heating pad for recovery and returned to their dams after waking from surgery. Sham-operated rat pups were treated identically but did not undergo $\mathrm{CCl}$. The four groups ( $n=6 /$ group) of rat pups were randomized between sham, mild, moderate, and severe injury.

\section{2 | MRI and analysis}

In the present study on TBI severity in the developing rodent, we elected not collect pre-injury baseline MRI due to the potential negative effects of repeated anesthetic exposure as have been reported (Bajwa et al., 2019). MRI was performed at $6 \mathrm{hr}$ (0.25 days), 1, and 3 days after $\mathrm{CCl}$. Rat pups were lightly anesthetized using isoflurane ( $<1 \%$ maintenance) and body temperature was maintained at $37 \pm 1^{\circ} \mathrm{C}$ using a thermostat-controlled heated water cushion. MR data were collected on a Bruker Advance 4.7T MRI using a fourchannel quadrature radio frequency coil (Bruker Biospin, Billerica, MA). We evaluated two key MRI data types: (a) T2-weighted imaging (T2WI) for edema development and formation, and (b) susceptibility-weighted imaging (SWI) for localization of extravascular blood. The T2 sequence had the following parameters: TR/TE (time to repetition/echo time) $=3,278 \mathrm{~ms} / 20 \mathrm{~ms}$, matrix $=256^{2}$, field of view $(F O V)=2.3 \mathrm{~cm}, 2$ averages and 6 echos. The SWI sequence was acquired using the following parameters: TR/TE $=57.8 / 20 \mathrm{~ms}$, flip angle $=20$ degrees, matrix $=256^{2}, \mathrm{FOV}=2.3 \mathrm{~cm}, 23 \mathrm{~mm}$ slab thickness with 48 partitions. Total imaging time was $51 \mathrm{~min}$.

$\mathrm{T} 2$ relaxation rates were determined for each pixel and T2 maps were generated using in-house Matlab routines (Matlab, Natick, MA; RRID:SCR_001622) as described previously (Obenaus et al., 2007). T2 images were analyzed using regions of hyperintensity to delineate the spatial development of the lesion volume (edema) using 3D imaging software (Amira, Mercury Computer Systems, San Diego, CA; RRID:SCR_014305) to extract hyperintense lesion volumes, ipsilateral and contralateral hemispheric brain volumes. SWI magnitude and phase data sets were mapped using Spin image processing software (The MRI Institute for Biomedical Research, Detroit, MI). In some animals, the SWI displayed significant susceptibility artifacts due to blood or bone/air interfaces precluded accurate data analysis and were excluded from the study, leading to smaller replicates at some time points. Lesion volume analysis in Amira included (hypointensities) data from SWI images. Blood lesion volumes were calculated in the same manner except SWI was used to determine blood volume. The blood/edema volumes were divided by the sum of both hemisphere volumes to correct for individual brain size differences.

The number of animals in each group at the onset of the experiments was $n=6$, which in our previous MRI studies resulted in sufficient statistical power. However, the number of replicates for the mild at the final experimental time point ( 3 day) was only $n=3$ for the mild as a number of pups were sacrificed at 1 day post-TBI for histological assessments. SWI data were examined for putative susceptibility artifacts due to air-bone interfaces or poor SW image reconstruction; this resulted in $n<6$ for some time points.

\section{3 | Histology}

After imaging, all animals were transcardially perfused with $4 \%$ paraformaldehyde and put in 30\% sucrose in phosphate-buffered saline (PBS) for cryoprotection before freezing and cutting on a cryostat (Leica Biosystems, Buffalo Grove, IL). All staining was performed on $30 \mu \mathrm{m}$ sections centered at the lesion site from 3 days post-jTBI tissues. Prussian blue staining was performed as previously described to detect extravascular blood within the tissue (Baghchechi et al., 2016; Donovan et al., 2012; Obenaus et al., 2011). Degenerating neurons were detected using fluoro-jade B (FJB; Millipore, Temecula, CA) staining on thawed tissue sections (Schmued \& Hopkins, 2000). The number of damaged cells (FJB+) within the tissue was quantified using ImageJ software (ITCN plugin, U.S. National Institutes of Health, Bethesda, MD; RRID:SCR_003070). In four randomly selected animals from each group, two adjacent sections from each rat pup were imported into ImageJ and converted to 8 bit and inverted. Four primary regions of interest (ROIs) including lesion, perilesion, ipsilateral and contralateral CC were delineated on the two adjacent sections. The number of FJB + cells was quantified by averaging the number of positive cells from the two sections. To determine the number of FJB + neurons in the ipsilateral cortex, the number of cells in lesion and perilesion was summed. The total number of FJB + cells for each pup was calculated by combining all four ROIs.

\section{4 | Statistics}

Explicit power analysis was not undertaken but previous published studies in similar groups of animals and in adult studies using MRI have found that $n=6$ is typically sufficient for statistical reporting (Donovan et al., 2012; Fukuda et al., 2013; Igarashi et al., 2007; Jullienne et al., 2018; Obenaus et al., 2007; Rodriguez-Grande et al., 2018). All juvenile animals and groups were randomized. All analyses were conducted in a blinded fashion without knowledge of groups or treatment. Using GraphPad Prism, (Version 8.01, San Diego, CA, 
RRID:SCR_002798, MRI blood, edema volume data, and Fluorojade $B$ staining were analyzed by mixed effects one-way analysis of variance (ANOVA) with post hoc Tukey's test. Linear regressions were performed over the full extent of the axis's using SigmaPlot (Systat Software Inc., San Jose, CA; RRID:SCR_003210), as shown in Figure 6. An $\alpha$-level of 0.05 was used for all statistical significance tests. All data are expressed as the mean \pm SEM.

\section{3 | RESULTS}

All rat pups survived the jTBI procedure independent of the injury severity. Unless noted otherwise, all volumes are normalized to whole brain volume (BV).

\section{1 | Multimodal MRI confirms jTBI severity at $6 \mathrm{hr}$ post-injury}

The lesion size and attendant edema was clearly visible on the T2WI as early as $6 \mathrm{hr}$ post-injury and increased with increasing injury severity (Figure 1, top row). In the more severe instances of jTBI, there was the appearance of hypointense pixels that reflected the presence of extravascular blood. SWI was used to visualize and quantitate the volume of extravascular blood (Figure 1, middle row). As can be readily appreciated, the SWI very clearly revealed the presence of blood in the tissues even in mild jTBI pups, with increasing blood volume as a function of severity. Three-dimensional reconstructions from SWI illustrated the increasing lesion volume associated with the increasing depth of cortical compression (Figure 1, bottom row). In severe jTBI, there was hippocampal edema in all of the animals studied (100\%), compared to $60 \%$ in moderate and $0 \%$ in mild jTBI. There was no visible lesion due to the craniotomy in sham pups on T2WI and SWI.

\section{2 $\mathrm{CCl}$ depth increases early edema formation}

Increased T2-hyperintensities spanning the entire cerebrum were used to measure edema formation temporally to quantify edema lesion volume. Shams did not have any detectable edema volumes across any time point we examined. Mild jTBI $(1.0 \mathrm{~mm}$ depth) resulted in $0.11 \% \pm 0.04$ edema volume of the total brain volume at $6 \mathrm{hr}$, which was almost undetectable at 3 days $(0.004 \%$ \pm 0.01 ) (Figure 2a,c,d). Moderate jTBI (1.5 mm depth) resulted in edema lesion volumes of $0.51 \% \pm 0.04$ at $6 \mathrm{hr}$ that progressively decreased and at 3 days the edema volume had decreased $73 \%$ $(0.15 \% \pm 0.03)$ compared to 1 day $(p<0.05)$ (Figure $2 a, c, d)$. Severe jTBI ( $2 \mathrm{~mm}$ depth) rat pups exhibited the largest edema volumes at all time points, with $0.83 \% \pm 0.09$ at $6 \mathrm{hr}, 0.60 \% \pm 0.08$ at

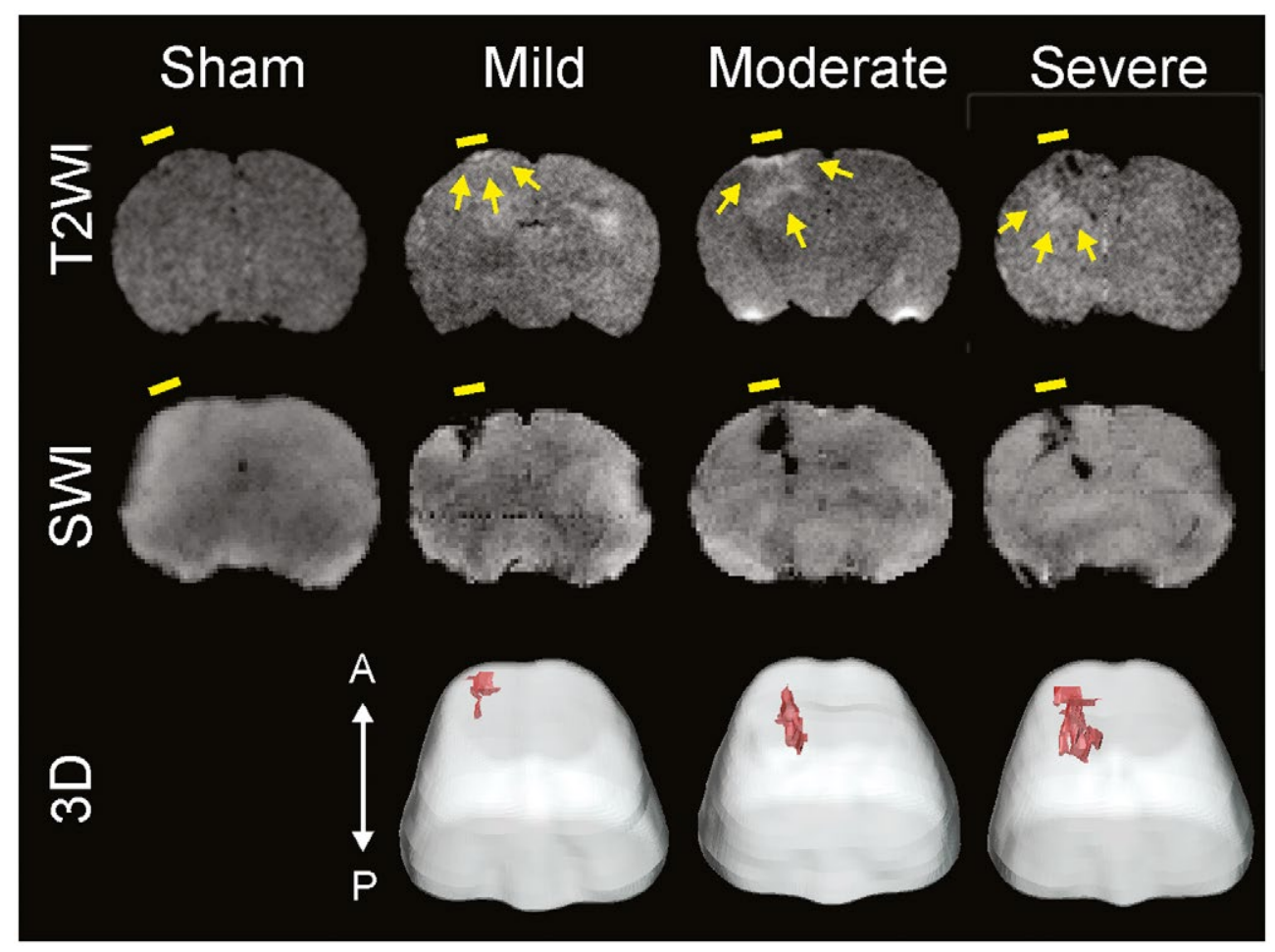

FIG URE 1 Multimodal MRI assessments of traumatic brain injury at $6 \mathrm{hr}$ after graded jTBI severity. Noninvasive T2WI, SWI, and T2WI lesion-derived 3D reconstructions illustrated that with increased injury severity there were increased lesion volumes with altered lesion characteristics at $6 \mathrm{hr}$. The site of injury can be visualized on both T2WI for edema (hyperintense, yellow arrows) and blood (hypointense) in the brain of jTBI pups. SWI MRI was optimized to report the presence of blood), hypointense). There were no visible alterations due to the craniotomy in all groups (yellow line). SWI 3D reconstruction of the injured brain at $6 \mathrm{hr}$ post-injury illustrated increasing lesion volume (red) with increasing TBI severity within the brain parenchyma (white). ( $\mathrm{A}-\mathrm{P}=$ anterior to posterior) 

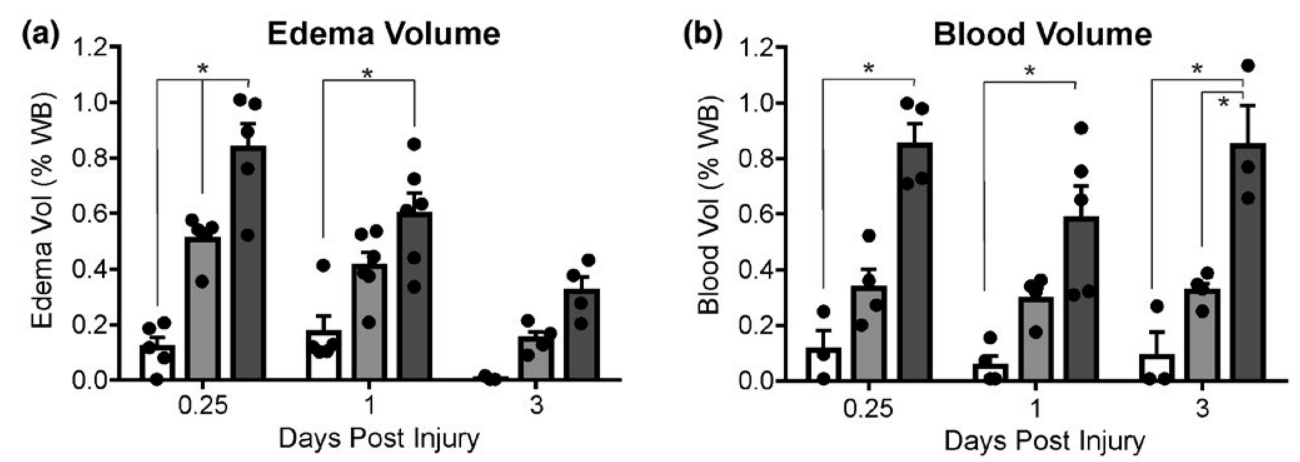

$\square$ Mild $\square$ Moderate $\square$ Severe
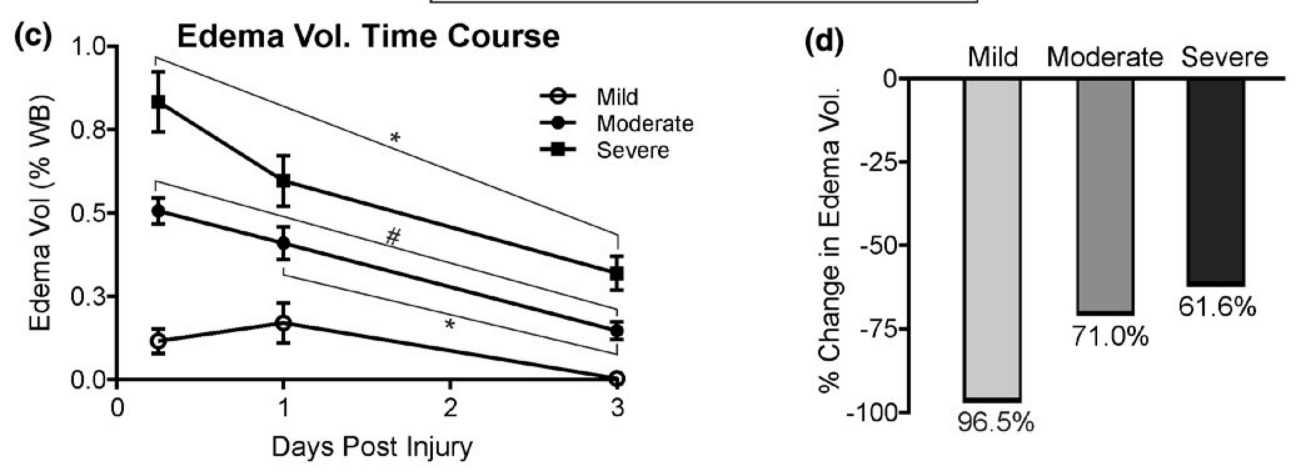

FIGURE 2 Edema and blood volumes increase with jTBI severity. (a) Edema volumes quantified from T2WI reported a significant interaction between severity (mild, moderate, and severe) and time point $(0.25,1$, and 3 days) following injury (two-way ANOVA, $p<0.05$ ). Shams $(n=5)$ had no detectable edema volumes at any time point (data not shown). Mild jTBI $(n=5)$ resulted in edema volume at 0.25 days ( $6 \mathrm{hr}$ ) that was $0.11 \% \pm 0.04 \%$ of brain volume, which was decreased to $0.004 \% \pm 0.01$ at 3 days. Edema volume was $0.51 \% \pm 0.04$ of the brain volume after moderate jTBI $(n=5)$ at 0.25 days. At 3 days, the edema volume was $0.15 \% \pm 0.03$, which had a $73 \%$ decrease compared to 0.25 days $(p<0.05)$. Severe $\mathrm{jTBI}(n=6)$ rat pups started with the highest percentage of edema volume at $0.83 \% \pm 0.09$ at $6 \mathrm{hr}$. Similar to mild and moderate jTBI pups, severe edema volume decreased $61 \%$ by 3 days to $0.32 \% \pm 0.05$ ( $p<0.05$ ). (b) Blood volumes revealed a significant difference between each group at each time point (two-way ANOVA, $p<0001$ ) but no time-dependent effects. Sham surgery resulted in a barely detectable blood volume $(0.007 \% \pm 0.001 \%)$ at 0.25 days and which was undetectable at 1 or 3 days post-surgery (data not shown). Mild jTBI resulted in a blood volume of $0.08 \% \pm 0.07 \%$ at 0.25 days with a small reduction of $38 \%$ at 1 day (compared to 0.25 days) and reported a final 3 -day volume of $0.09 \% \pm 0.15 \%$. Moderate jTBI resulted in a sustained blood volume of $0.33 \% \pm 0.09 \%, 0.29 \%$ $\pm 0.07 \%$, and $0.32 \% \pm 0.02$ at $0.25,1$, and 3 days post-injury, respectively. Severe jTBI pups exhibited the highest blood volume of $0.85 \%$ $\pm 0.20 \%$ at 0.25 days that then decreased to $0.58 \% \pm 0.12$ at 1 day, with a final blood volume of $0.85 \% \pm 0.14 \%$ at 3 days post-jTBI. (c) The temporal evolution of edema volume changes in mild, moderate, and severe jTBI pups. In each severity, there was an almost uniform rate of edema reduction over the 3-day time course. All data were assessed using a mixed effects ANOVA and missing data points were modeled. $\left(1 d^{*} p=0.023, \# p=0.029,3 d^{*} p=0.054\right)$. (d) The relative change in edema volume change between 0.25 and 3 days illustrates that edema is virtually resolved in mild jTBI pups but less so with increasing severity. (see $\mathrm{C}$ for statistical significance)

1 day, and $0.32 \% \pm 0.05$ at 3 days (Figure 2a,c,d). Similar to mild and moderate jTBI pups, severe edema volume decreased by $61 \%$ $(p<0.05)$ over the course of the 3 days. Lastly, there was a significant severity group and time interaction $(p<0.04)$. Thus, jTBI severity illustrates the graded edema response, whose volumes significantly decreased over 3 days.

The temporal nature of edema resolution in mild, moderate, and severe jTBI was assessed (Figure 2c). Given the relatively minimal edema volumes in the mild jTBI pups, there was no significant difference in edema volumes over the course of 3 days after injury, where at 3 days the edema volume was negligible. With increasing severity, there was an $~ 30 \%$ increase in edema volume at $6 \mathrm{hr}$ after TBI induction (Figure 2c). In the moderate jTBI pups, there was a significant linear decrease in edema volumes over the 3-day observation pe$\operatorname{riod}(p<0.05)$ and a significant decrease from 1 to 3 days post-injury $(p<0.05)$ (Figure 2c). With the increased lesion and edema variability in the severe jTBI group, a significant reduction was observed only between 0.25 and 3 days $(p<0.054)$ (Figure $2 c)$. The relative change in edema volume is plotted in Figure $2 d$, where there is a severity-dependent reduction in the amount of edema volume. It is important to note blood volumes (Figures $2 b$ and $3 a, b$ ) may contribute to the relative decrement in edema resolution as a function of increasing severity due to increasing blood volumes.

\section{3 | Blood volume stays relatively unchanged after 3 days}

The volumes of hypointensities on SWI (Figure 1) were quantified as a measure of blood volume, as a consequence of jTBI. Sham surgery resulted in minimal blood appearance on the pial surface $(0.07 \% \pm$ 

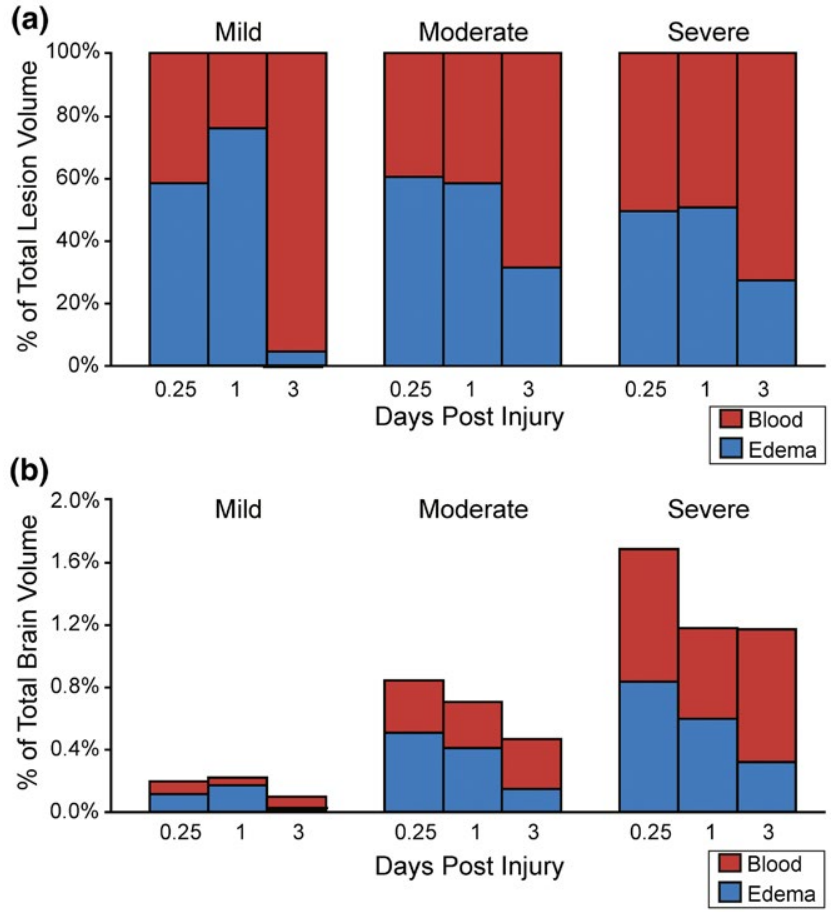

FIGURE 3 Blood and edema lesion composition after graded jTBI. (a) Blood and edema within the lesion volume revealed that at 0.25 days, the mild jTBI lesion volume was comprised of $\sim 60 \%$ edema and $\sim 40 \%$ blood, As edema resolved at later time points, blood constituted the majority of the lesion volume in mild jTBI. In contrast, the proportion of blood in moderate and severe jTBI was relatively constant at the early time points, but constituted $\sim 70 \%$ of the total lesion as edema resolved by 3 days. (b) Comparison of blood and edema volumes relative to total brain volumes showed that blood volume was increased with increasing severity (mild, moderate, and severe) but remained constant over the 3-day period. As shown, edema in all jTBI severities was highest at 0.25 days but decreased by $~ 50 \%$ at 3 days post-TBI. At 0.25 days post-jTBI, total lesion volumes (blood + edema) relative to total brain volume after mild, moderate, and severe jTBI were $0.19 \%$, $0.84 \%$, and $1.68 \%$, respectively

$0.01,6 \mathrm{hr}$ ) which disappeared by 1 day post-injury (0.00\%). Whereas there was no blood visible on T2 in mild jTBI, we unexpectedly found that SWI identified blood volume of $0.08 \% \pm 0.07$ at $6 \mathrm{hr}$ (Figure $2 \mathrm{~b}$ ). At 1 day in the mild jTBI pups, blood volume decreased by $38 \%$ with a final blood volume of $0.09 \% \pm 0.15$ at 3 days, and there was no significant difference between blood volumes at any time point. A similar pattern of relatively minor changes in blood volumes was also observed in both moderate and severe jTBI. In moderate jTBI, at $6 \mathrm{hr}$ the resulting blood volume was $0.33 \% \pm 0.09$, at 1 day it was $0.29 \%$ \pm 0.07 , with a 3 -day volume of $0.32 \% \pm 0.02$. Similar to edema lesion volumes, severe $\mathrm{jTBI}$ pups had the highest blood volumes at $0.85 \%$ \pm 0.20 at $6 \mathrm{hr}$ that then decreased at 1 day $(0.51 \% \pm 0.35)$ and at 3 days exhibited a final blood volume of $0.85 \% \pm 0.14$. We observed a significant difference between each injury severity group at each time point $(p<0.001)$, but no significance was noted across time within each severity group. The presence of blood on SWI is a good reporter for injury severity as it is clearly visible even at early time points, particularly mild TBI.

\subsection{Spatial and temporal lesion composition}

To evaluate the spatial and temporal relationship between edema and blood volumes after $\mathrm{jTBI}$, we compared the overall lesion composition (blood, edema) as a percentage of the total lesion. We found that independent of jTBI severity, lesion volume was comprised of $\sim 60 \%$ edema and $\sim 40 \%$ blood at $6 \mathrm{hr}$ post-injury (Figure 3a). This proportion remained relatively the same across all groups until 3 days, where mild jTBI lesion was composed of $95.7 \%$ blood at 3 days. This apparent increase in blood composition of lesion volume was predominantly due to the resolution of edema in the mild jTBI rat pups. The proportion of blood from moderate and severe jTBI pups stayed below $70 \%$ of the total lesion as the edema resolved at a much slower rate during the 3-day time course.

We also examined absolute blood volumes as a percentage of whole brain volumes which illustrated the importance of blood deposition in the brain at $6 \mathrm{hr}$. Regardless of edema volume at $6 \mathrm{hr}$, blood volume was indicative of jTBI severity. Blood volume at $6 \mathrm{hr}$ was highest after severe jTBI and lowest after mild jTBI (Figure 3b). Taken together, blood volume as a function of brain volume is an excellent imaging-based predictor of severity and the rate at which edema resolves at 3 days after injury.

\subsection{Blood deposition in the CC after severe jTBI}

We observed on histology that there was considerable blood deposition in the CC in severe jTBI. We carried out Prussian blue staining for extravascular blood within the impacted hemisphere at 3 days post-jTBI. Prussian blue staining demonstrated increased blood deposition within the ipsilateral cortex and CC with increasing jTBI severity (Figure 4a). Blood deposition appeared concentrated at the impact site in mild jTBI group (Figure 4a,b), while in moderate and severe jTBI there was increased blood deposition that spread from the cortex to the CC (Figure 4a-c). Prussian blue positive staining was also observed in the contralateral $\mathrm{CC}$ in severe jTBI, but not detected in mild nor moderate jTBI (Figure 4b). There was a clear progressive increase in the number of jTBI animals that exhibited extravascular blood in Prussian blue staining with increasing severity (Figure 4c).

\section{6 | Degenerating neurons increase with increasing severity}

Given the proximity of the $\mathrm{CC}$ to the impact zone, we measured degenerating cells in the cortex and CC using FJB. Within the cortex we found a substantial number of FJB + cell bodies at the site of the impact suggesting ongoing cellular degradation (Figure 5a). At higher magnification, it is clear that degenerating cells included both cell bodies in the cortex and attendant axonal processes 
(a) Mild

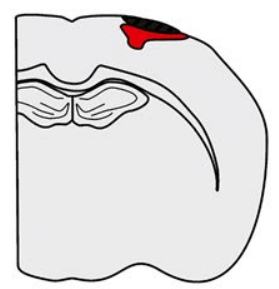
Moderate
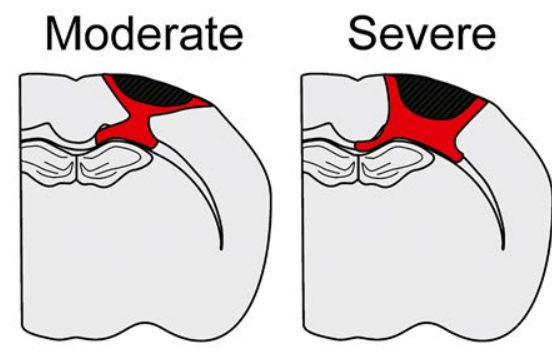

(b)

Severe

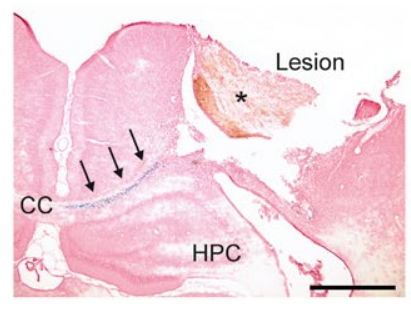

Ipsilateral

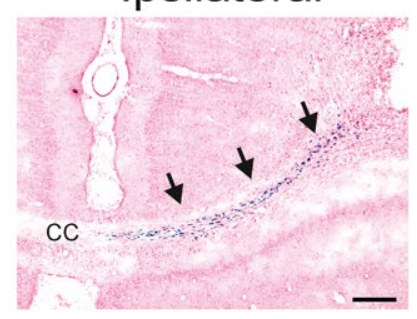

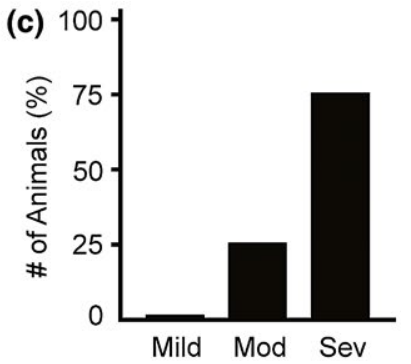

Contralateral

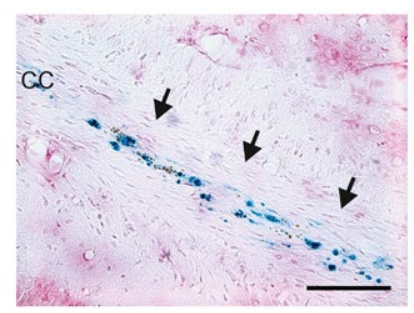

FIG URE 4 Blood deposition at 3 days after graded jTBI. (a) Representative average tissue schematics of mild, moderate, and severe jTBI at 3 days post-injury illustrating the extent of cavitation (black) and blood deposition (red) within the cortex and CC (moderate, severe). (b). Prussian blue staining in severe jTBI was only detected in the ipsilateral (arrows) and contralateral CC at the site of maximal lesion volume. Residual extravascular blood could also be observed in the pan-necrotic cortical tissues $\left({ }^{*}\right)$. HPC = hippocampus. (note: contralateral image is $2 \mathrm{X}$ relative to the ipsilateral to illustrate blood deposition) (c) The number of animals with Prussian blue staining in the contralateral CC after mild jTBI was $0 \%, 25 \%$ in moderately injured pups whereas $75 \%$ of severe jTBI rat pups exhibited extravascular blood in the white matter. Scale bars: Severe $=1 \mathrm{~mm}$; Ipsilateral, contralateral $=100 \mu \mathrm{m}$

in the CC (Figure 5b, left panel). Similarly, cell bodies were observed within the cingulum and CC (Figure $5 \mathrm{~b}$, right panel). In the lesion and perilesion regions, there was an $\sim 3$-fold increase in $\mathrm{FJB}+$ cells after moderate jTBI compared to mild with a further $\sim 2$-fold increase in severe compared to moderate jTBI (Figure 5a,c; $p<0.001)$. This profile was also observed when FJB + cells were quantified in the ipsilateral CC $(84 \pm 32$ mild vs. $145 \pm 21$ moderate vs. $217 \pm 31$ severe, $p<0.032$ ). Total fluoro-jade cell counts revealed an almost doubling of degenerating cells from mild to moderate to severe $(179 \pm 25$ mild vs. $481 \pm 79$ moderate vs. $837 \pm 72$ severe, $p<0.001$ ).

\section{7 | Relationship between cell death and MRI-derived blood and edema}

We further explored if the number of degenerating cells was related to the volume of edema or extravascular blood. When total $\mathrm{FJB}+$ cells (cortex $+\mathrm{CC}$ ) were combined, we observed significant strong correlations to blood volumes at $6 \mathrm{hr}$ and 1 days post-injury that declined by 3 days (Figure 6a). With edema, a significant correlation was present with FJB + cells at $6 \mathrm{hr}$, but no significant relationship was found at later time points (Figure 6b). Cortical FJB + cells at 3 days post-injury were significantly correlated to blood volume at $6 \mathrm{hr}, 1$ and 3 days (Figure 6c) suggesting that extravascular blood substantially contributed to cellular degeneration in jTBI. In contrast, neither CC nor cortical FJB + cells were correlated to edema volumes (Figure 6d).

\section{4 | DISCUSSION}

In this preclinical study, we sought to extensively characterize noninvasively three different grades (mild, moderate, and severe) of jTBI in the PND 17 rats using a $\mathrm{CCl}$ model during the acute injury phase. The novel findings of this study are: (a) a multimodal neuroimaging strategy for assessment of edema and extravascular blood could stratify injury severity even for mild-TBI, (b) early blood volumes (6 hr) were prognostic for severity, (c) lesion composition (percentage of blood and water content) was similar over the first day between injury severities, independent of the size of the lesion, (d) evidence of bleeding into the CC on the ipsilateral and contralateral hemispheres, and (e) degenerating cells within the cortex of the impacted hemisphere were significantly correlated with blood volumes over the 3-day experimental time course, while edema volumes were not.

Preclinical models, including those for TBI, are often criticized for not recapitulating the clinical and human manifestations of disease. The model(s) discrepancy has been cited as basis for lack of success of the transfer of the new drugs from the bench to the bedside (Hyman et al., 2017). While relative standardization for adult models of TBI exist, for example, $\mathrm{FPI}$ and $\mathrm{CCl}$, there is virtually no such consistency present for models of juvenile TBI (Obenaus et al., 2012b; Semple, Carlson, \& Noble-Haeusslein, 2016). Indeed efforts by the National Institutes of Health in generating common data elements in animal models are a first step forward in assuring comparison of data between research laboratories (Manley et al., 2017). Such consistency or standardization not only applies to induction of 

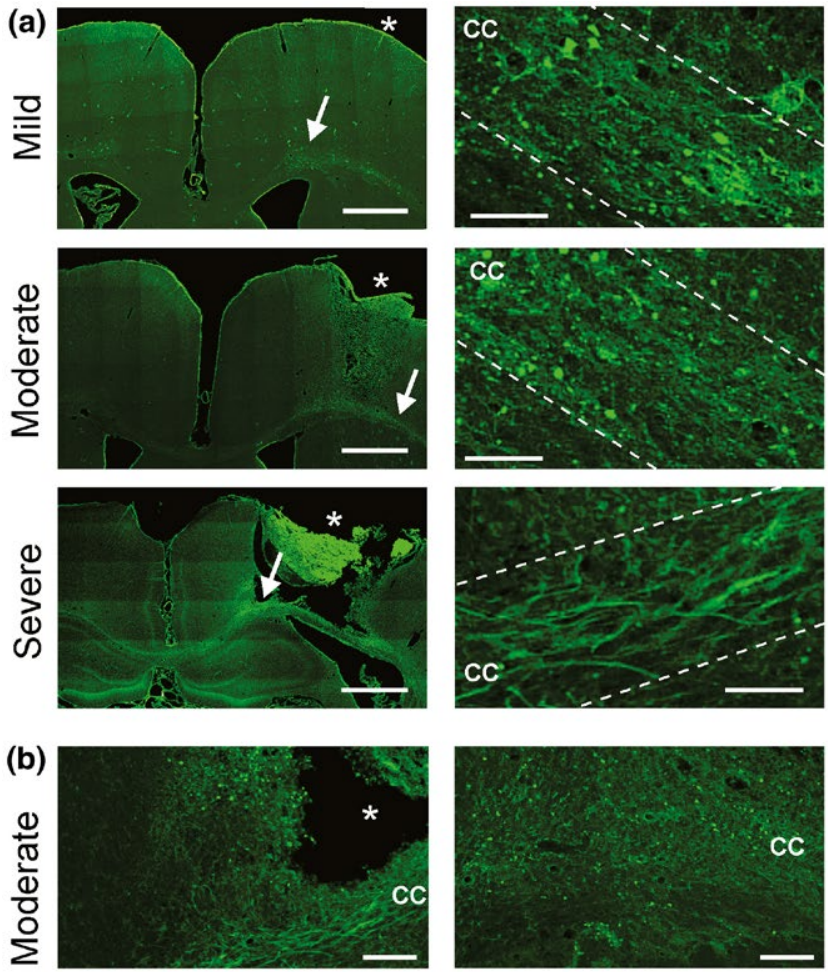

(c)
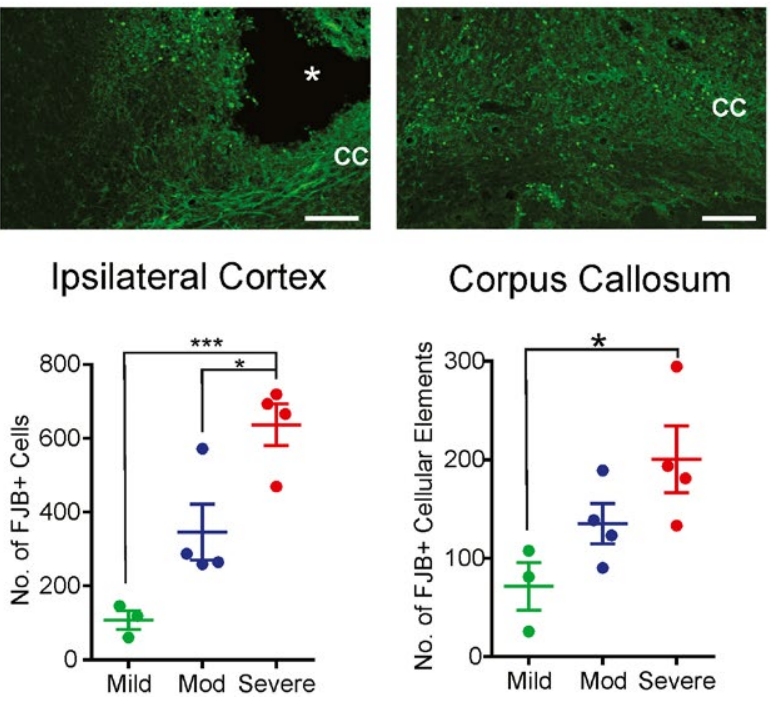

Corpus Callosum

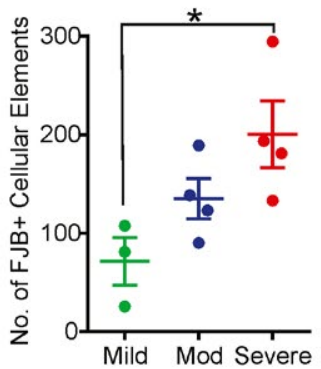

FIGURE 5 Cell death at 3 days after graded jTBI. (a) Cell death at 3 days post-injury was assessed using fluoro-jade $B$ (green) after mild, moderate, and severe jTBI. Increased fluorojade $\mathrm{B}$ positive cells were observed in the cortex and $\mathrm{CC}$ within increasing jTBI severity (left panel). At higher magnification (right panel) positive staining was observed in cells at all injury severities (mild, moderate, and severe). In the CC, the appearance of aggregated products in mild and moderate were observed with abnormal axonal elements seen in severe jTBI. Scale bars: left panel $=1 \mathrm{~mm}$, right panel $=50 \mu \mathrm{m}$. (b) Higher magnification of degenerating cells in the cortex and $\mathrm{CC}$ where neuronal and axonal elements are fluoro-jade B positive. In addition, other cell types such as astrocytes are also likely degenerating. Scale bars: $100 \mu \mathrm{m}$. (c) Quantification of degenerating cells revealed significant increases with increasing jTBI severity in the ipsilateral cortex and CC. $\left({ }^{*} p<0.05,{ }^{* * *} p<0.001\right)$

traumatic injury but also in subsequent outcome measures (histology, behavioral, etc.; (Siebold et al., 2018)).

Neuroimaging is increasingly being used in clinical and research studies of TBI with CT often being used as the initial screening tool for hemorrhage assessment and injury severity (Gardner et al.,
2018). T2WI is well known to assess edema and provides clear measures of edema (Obenaus \& Badaut, 2017). While clinically, edema volumes are not utilized routinely, in animal models of TBI the extent of edema is useful to monitor the progression of intervention (RodriguezGrande et al., 2018). SWI (and its variants) are now also used clinically and it have been shown to be very sensitive to extravascular blood following TBI in both adult and juvenile populations (Haacke et al., 2004; Tong et al., 2008). To the best of our knowledge, this is the first study to use SWI acutely in a juvenile rat model of TBI. We found that extravascular blood was present in all forms of severity in our $\mathrm{CCl}$ model of jTBI. The deposition of blood was predominately within the cortex at the impact zone (Figures 1 and 4) but as severity increased, elevated blood deposition was observed more frequently at distance from the injury site (Figure 4). There was increased bleeding in the CC following moderate and severe jTBI compared to that seen in adult models, a finding confirmed in human studies (Sarkar et al., 2014). The juvenile brain is still undergoing significant development and structurally may be more vulnerable to traumatic injury, as we observed here (Giza, Mink, \& Madikians, 2007). While the CCl model is relatively invasive, even emerging models of mild pediatric TBI with a closed head injury approach have reported the appearance of cortical hemorrhage using SWI (Rodriguez-Grande et al., 2018). Use of SWI is relatively atypical in preclinical model characterization and our findings along with others would suggest that this imaging modality would be useful for monitoring the appearance of hemorrhage after jTBI, particularly in the context of severity.

Another novel finding of our study was that lesion composition (edema or blood) was relatively constant in each injury severity over the first-day post-injury. It is clear that in human studies there is a heterogeneity in the appearance of blood after mild, moderate, or severe TBI (Isokuortti et al., 2018). Clinically after severe pediatric TBI, subarachnoid hemorrhage has been found to be positively associated with edema which leads to poor shortand long-term outcomes (Hochstadter, Stewart, Alharfi, Ranger, \& Fraser, 2014). While no studies have examined the relationship between hemorrhage and edema in $\mathrm{jTBI}$, we suggest that the degree of extravascular blood is related to the level of edema (Figure 3b). This is supported by the relationship between cell death and blood deposition (Figure 6c). The blood/edema association in all likelihood ultimately leads to poor outcomes even when edema resolves. Support for this hypothesis was our finding that degenerating cells at 3 days post-injury in the cortex significantly correlated to the volume of blood in the brain (Figure 6). Zhao and colleagues using a murine model of moderate TBI reported that cell death was initially less than that of adult mice at early time points (<1 day) (Zhao, Wang, Gao, \& Chen, 2018). However, at 1-3 days there were extensive FJB + cells within the cortex, and hippocampus. In addition, while blood volumes were not quantified in adult mice and rats the presence of hemorrhage in the cortex resulted in behavioral decrements (Yu et al., 2009).

Our study is unique in quantifying the relation between iron deposition (Prussian blue, SWI) and cell death (FJB + cells) in a jTBI model. Other studies have qualitatively described cell death after 

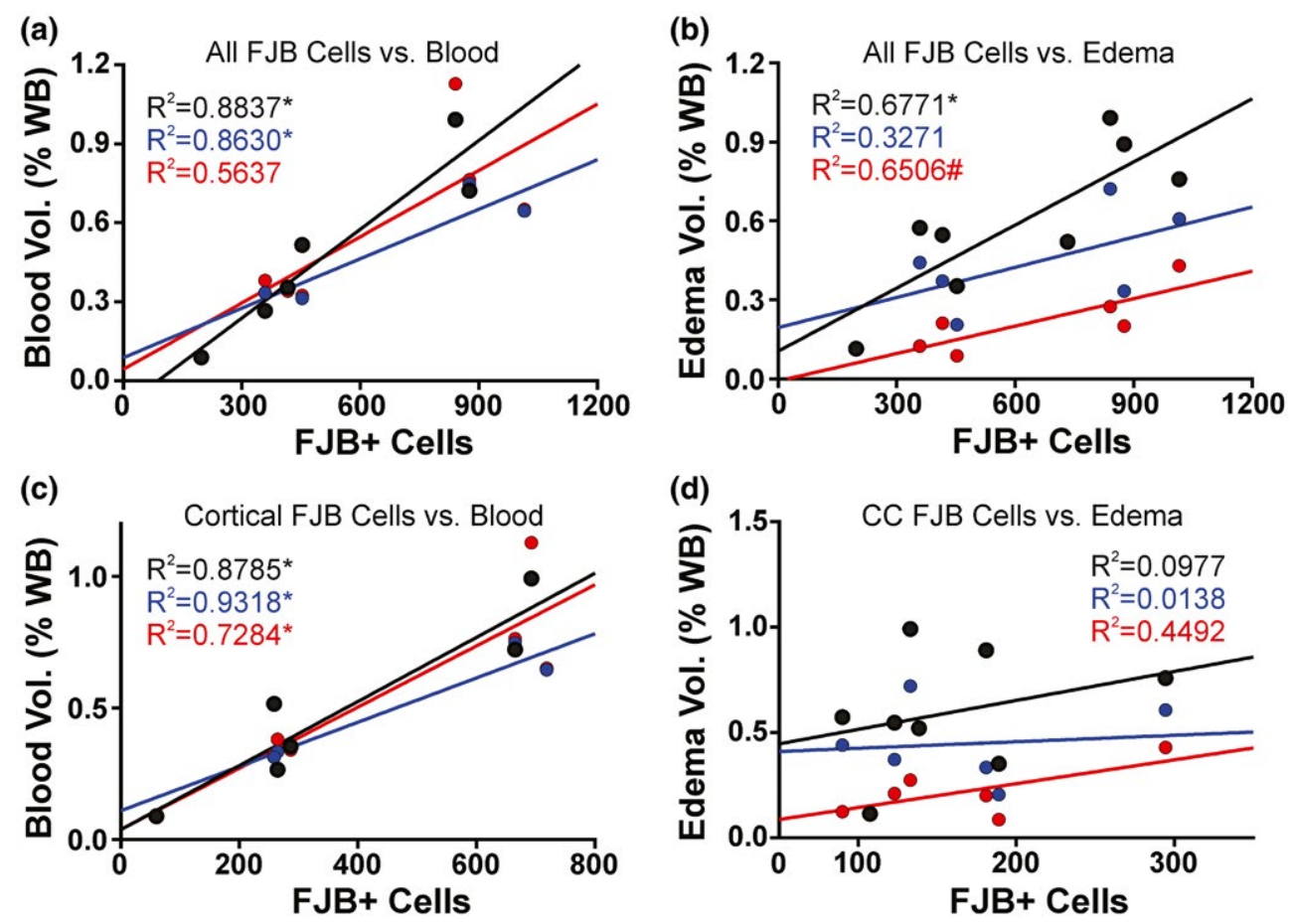

$$
0.25 d \rightarrow 1 d \rightarrow 3 d
$$

FIGURE 6 Relationship between cell death and blood and edema volumes. (a) A significant relationship between all fluoro-jade B positive $(\mathrm{FJB}+)$ cells (cortex and $\mathrm{CC}$ combined) and blood volumes was evident at 0.25 and 1 days post-injury but not at 3 days ( ${ }^{*}$ indicates significance at $p<0.05$ ). (b) A similar relationship was also found with all FJB + cells compared to total edema volumes (CC and cortex). At 3 days, there was a trending effect $(\# p=0.053)$. (c) Cortical regions predominately contributed to the significant relationships as seen when correlations were made of cortical FJB + cells and blood volume. A virtually identical relationship was seen with cortical FJB + cells and edema volumes (data not shown). (d) The FJB + cells in the CC did not contribute significantly by edema specifically at 0.25 and 1 days post-injury nor blood (data not shown) volumes

TBI in adult rats (Hawkins et al., 2013; Immonen, Kharatishvili, Grohn, Pitkanen, \& Grohn, 2009; Kharatishvili et al., 2009) and swine (Manley et al., 2006) but this study is the first to do so in the immature brain. The addition of clinically-relevant MR imaging for assessment of blood and edema is also novel and provides the basis for future studies evaluating interventions.

Therapeutically, there are no targeted interventions for blood or edema development in jTBI. Edema can be relieved by several strategies experimentally and our own published work has shown that modulating the brain water channel, acquaporin-4 (AQP4) early after TBI can reduce edema and improve outcomes (Badaut, Ashwal, \& Obenaus, 2011; Fukuda et al., 2013). In adult rats, the iron chelator, deferoxamine, was able to reduce brain iron (blood) and ultimately improve outcomes after TBI (Zhang et al., 2013). Potts and colleagues also note in a survey of therapeutic strategies for the immature brain that iron accumulation potentially accumulates more rapidly in the young brain compared to the adult brain and posit that iron chelation in children with TBI is a therapeutic target (Potts et al., 2006). However, this has to our knowledge not been explicitly tested.

A number of limitations of the present study are noted. Firstly, no behavioral tests were undertaken within the current study. We acknowledge that even rudimentary behavioral tests for the immature rat would have been beneficial, but the focus was to assess the temporal evolution of injury severities (mild, moderate, and severe) with respect to blood and edema volumes. Secondly, we only used the $\mathrm{CCl}$ model in this study which limits its translation to other juvenile models, such as FPI. However, moderate FPI at PND 17, similar to our study revealed hemorrhage, seizures, and increased apnea in relation to adolescent (PND 35) and adult rats (Rowe, Harrison, Ellis, Adelson, \& Lifshitz, 2018). The temporal evolution of neuroimaging findings is likely to be different in the immature brain and is likely dependent upon the TBI model utilized, as has been reported in adult TBI (Obenaus et al., 2007). Lastly, we did not explicitly evaluate how the immature female responds to graded injury severity. The jTBI was induced prior to hormonal maturation which could result in developmental-related modification of outcomes, as we have reported in adult TBI (Jullienne et al., 2018). The effects of juvenile TBI and long-term outcomes on gender warrant future study.

In conclusion, we provide convincing noninvasive MRI assessments of the relationships between post-injury abnormal blood accumulation and the severity of jTBI. Extravascular blood was the principal determinant of cellular death, particularly at early post-injury time points. Clinically relevant imaging also illustrated the need for MR imaging methods sensitive to blood deposition, as we found SWI discernable blood even in mild jTBI. This study provides the basis to evaluate therapeutic compounds to limit blood and edema 
to mitigate cell death and improve long-term outcomes. It also confirms the need to study the pediatric brain after trauma which is unique in its response to injury relative to the adult brain.

\section{DECLARATION OF TRANSPARENCY}

The authors, reviewers, and editors affirm that in accordance with the policies set by the Journal of Neuroscience Research, this manuscript presents an accurate and transparent account of the study being reported and that all critical details describing the methods and results are present.

\section{ACKNOWLEDGMENTS}

A portion of this material was performed in the Loma Linda University School of Medicine Advanced Imaging and Microscopy Core (LLUSM AIM) that is supported by the National Science Foundation under Major Research Instrumentation, Division of Biological Infrastructure Grant No. 0923559 (Sean M Wilson) and the Loma Linda University School of Medicine.

\section{CONFLICT OF INTEREST}

The authors have no conflicts of interest.

\section{AUTHOR CONTRIBUTIONS}

All authors had full access to all the data in the study and take responsibility for the integrity of the data and the accuracy of the data analysis. Conceptualization, J.B., A.A., L.H., and A.O.; Methodology, A.A., L.H., and A.O.; Investigation, A.A and L.H.; Formal Analysis, A.A., L.H., and A.O.; Resources: J.B. and A.O.; Writing-Original Draft, J.B., A.A., L.H., and A.O.; Writing-Review \& Editing, J.B., L.H., and A.O.; Visualization, A.A. and A.O.; Supervision, J.B. and A.O.; Funding Acquisition, J.B. and A.O.

\section{DATA ACCESSIBILITY}

The data sets generated and analyzed during the current study are available from the corresponding author based on reasonable requests.

\section{ORCID}

Jerome Badaut (iD https://orcid.org/0000-0001-8475-0519

Arash Adami iD https://orcid.org/0000-0003-2741-6690

André Obenaus iD https://orcid.org/0000-0003-0081-6950

\section{REFERENCES}

Ajao, D. O., Pop, V., Kamper, J. E., Adami, A., Rudobeck, E., Huang, L., ... Badaut, J. (2012). Traumatic brain injury in young rats leads to progressive behavioral deficits coincident with altered tissue properties in adulthood. Journal of Neurotrauma, 29(11), 2060-2074. https://doi. org/10.1089/neu.2011.1883

Babikian, T., Merkley, T., Savage, R. C., Giza, C. C., \& Levin, H. (2015). Chronic aspects of pediatric traumatic brain injury: Review of the literature. Journal of Neurotrauma, 32(23), 1849-1860. https://doi. org/10.1089/neu.2015.3971

Badaut, J., Ashwal, S., \& Obenaus, A. (2011). Aquaporins in cerebrovascular disease: A target for treatment of brain edema? Cerebrovascular Diseases, 31(6), 521-531.

Baghchechi, M., Plaia, A., Hamer, M., Ghosh, N., Ashwal, S., \& Obenaus, A. (2016). Susceptibility-weighted imaging identifies iron-oxidelabeled human neural stem cells: Automated computational detection. Developmental Neuroscience, 38(6), 445-457. https://doi. org/10.1159/000455837

Bajwa, N. M., Lee, J. B., Halavi, S., Hartman, R. E., \& Obenaus, A. (2019). Repeated isoflurane in adult male mice leads to acute and persistent motor decrements with long-term modifications in corpus callosum microstructural integrity. Journal of Neuroscience Research, 97(3), 332-345. https://doi.org/10.1002/jnr.24343

Bauer, R., \& Fritz, H. (2004). Pathophysiology of traumatic injury in the developing brain: An introduction and short update. Experimental and Toxicologic Pathology: Official Journal of the Gesellschaft fur Toxikologische Pathologie, 56(1-2), 65-73.

DeKosky, S. T., \& Asken, B. M. (2017). Injury cascades in TBI-related neurodegeneration. Brain Injury, 31(9), 1177-1182. https://doi.org/10.10 80/02699052.2017.1312528

Dewan, M. C., Mummareddy, N., Wellons, J. C. 3rd, \& Bonfield, C. M. (2016). Epidemiology of global pediatric traumatic brain injury: Qualitative review. World Neurosurgery, 91(497-509), e491.

Di Battista, A., Soo, C., Catroppa, C., \& Anderson, V. (2012). Quality of life in children and adolescents post-TBI: A systematic review and meta-analysis. Journal of Neurotrauma, 29(9), 1717-1727. https://doi. org/10.1089/neu.2011.2157

Dikranian, K., Cohen, R., Mac Donald, C., Pan, Y., Brakefield, D., Bayly, P., \& Parsadanian, A. (2008). Mild traumatic brain injury to the infant mouse causes robust white matter axonal degeneration which precedes apoptotic death of cortical and thalamic neurons. Experimental Neurology, 211(2), 551-560. https://doi.org/10.1016/j. expneurol.2008.03.012

Donovan, V., Bianchi, A., Hartman, R., Bhanu, B., Carson, M. J., \& Obenaus, A. (2012). Computational analysis reveals increased blood deposition following repeated mild traumatic brain injury. Neurolmage: Clinical, 1(1), 18-28. https://doi.org/10.1016/j. nicl.2012.08.001

Fukuda, A. M., Adami, A., Pop, V., Bellone, J. A., Coats, J. S., Hartman, R. E., ... Badaut, J. (2013). Posttraumatic reduction of edema with aquaporin-4 RNA interference improves acute and chronic functional recovery. Journal of Cerebral Blood Flow and Metabolism: Official Journal of the International Society of Cerebral Blood Flow and Metabolism, 33(10), 1621-1632. https://doi.org/10.1038/ jcbfm. 2013.118

Gardner, R. C., Rubenstein, R., Wang, K. K. W., Korley, F. K., Yue, J. K., Yuh, E. L., ... Manley, G. T. (2018). Age-related differences in diagnostic accuracy of plasma glial fibrillary acidic protein and tau for identifying acute intracranial trauma on computed tomography: A TRACKTBI study. Journal of Neurotrauma, 35(20):2341-2350. https://doi. org/10.1089/neu.2018.5694

Giza, C. C., Mink, R. B., \& Madikians, A. (2007). Pediatric traumatic brain injury: Not just little adults. Current Opinion in Critical Care, 13(2), 143-152. https://doi.org/10.1097/MCC.0b013e32808255dc

Gotschall, C. S., Papero, P. H., Snyder, H. M., Johnson, D. L., Sacco, W. J., \& Eichelberger, M. R. (1995). Comparison of three measures of injury severity in children with traumatic brain injury. Journal of Neurotrauma, 12(4), 611-619. https://doi.org/10.1089/ neu.1995.12.611 
Haacke, E. M., Xu, Y., Cheng, Y. C., \& Reichenbach, J. R. (2004). Susceptibility weighted imaging (SWI). Magnetic Resonance in Medicine, 52(3), 612-618. https://doi.org/10.1002/mrm.20198

Haghbayan, H., Boutin, A., Laflamme, M., Lauzier, F., Shemilt, M., Moore, L., ... Turgeon, A. F. (2016). The prognostic value of magnetic resonance imaging in moderate and severe traumatic brain injury: A systematic review and meta-analysis protocol. Systematic Reviews, 5, 10. https://doi.org/10.1186/s13643-016-0184-x

Hawkins, B. E., Cowart, J. C., Parsley, M. A., Capra, B. A., Eidson, K. A., Hellmich, H. L., ... Prough, D. S. (2013). Effects of trauma, hemorrhage and resuscitation in aged rats. Brain Research, 1496, 28-35. https://doi.org/10.1016/j.brainres.2012.12.027

Hochstadter, E., Stewart, T. C., Alharfi, I. M., Ranger, A., \& Fraser, D. D. (2014). Subarachnoid hemorrhage prevalence and its association with short-term outcome in pediatric severe traumatic brain injury. Neurocritical Care, 21(3), 505-513. https://doi.org/10.1007/ s12028-014-9986-7

Hyman, S., Balice-Gordon, R., Brady, L., Bruijn, L., Conley, R., Farahany, N., ... Zorn, S. (2017). Therapeutic development in the absence of predictive animal models of nervous system disorders: Proceedings of a workshop. Washington, DC.

Igarashi, T., Potts, M. B., \& Noble-Haeusslein, L. J. (2007). Injury severity determines Purkinje cell loss and microglial activation in the cerebellum after cortical contusion injury. Experimental Neurology, 203(1), 258-268. https://doi.org/10.1016/j.expneurol.2006.08.030

Immonen, R. J., Kharatishvili, I., Grohn, H., Pitkanen, A., \& Grohn, O. H. (2009). Quantitative MRI predicts long-term structural and functional outcome after experimental traumatic brain injury. Neurolmage, 45(1), 1-9. https://doi.org/10.1016/j.neuroimage.2008.11.022

Isokuortti, H., Iverson, G. L., Silverberg, N. D., Kataja, A., Brander, A., Ohman, J., \& Luoto, T. M. (2018). Characterizing the type and location of intracranial abnormalities in mild traumatic brain injury. Journal of Neurosurgery, 129(6), 1588-1597. https://doi. org/10.3171/2017.7.JNS17615

Jullienne, A., Salehi, A., Affeldt, B., Baghchechi, M., Haddad, E., Avitua, A., ... Obenaus, A. (2018). Male and female mice exhibit divergent responses of the cortical vasculature to traumatic brain injury. Journal of Neurotrauma, 35(14), 1646-1658. https://doi.org/10.1089/ neu. 2017.5547

Kharatishvili, I., Sierra, A., Immonen, R. J., Grohn, O. H., \& Pitkanen, A. (2009). Quantitative T2 mapping as a potential marker for the initial assessment of the severity of damage after traumatic brain injury in rat. Experimental Neurology, 217(1), 154-164. https://doi. org/10.1016/j.expneurol.2009.01.026

Kurland, D., Hong, C., Aarabi, B., Gerzanich, V., \& Simard, J. M. (2012). Hemorrhagic progression of a contusion after traumatic brain injury: A review. Journal of Neurotrauma, 29(1), 19-31. https://doi. org/10.1089/neu.2011.2122

L. Wilson, M., Tenovuo, O., Mattila, V. M., Gissler, M., Celedonia, K. L., Impinen, A., \& Saarijärvi, S. (2017). Pediatric TBI in Finland: An examination of hospital discharges (1998-2012). European Journal of Paediatric Neurology, 21(2), 374-381. https://doi.org/10.1016/j. ejpn.2016.10.008

Levin, H. S., Wilde, E. A., Chu, Z., Yallampalli, R., Hanten, G. R., Li, X., ... Hunter, J. V. (2008). Diffusion tensor imaging in relation to cognitive and functional outcome of traumatic brain injury in children. Journal of Head Trauma Rehabilitation, 23(4), 197-208. https://doi. org/10.1097/01.HTR.0000327252.54128.7c

Liu, C., Li, W., Tong, K. A., Yeom, K. W., \& Kuzminski, S. (2015). Susceptibility-weighted imaging and quantitative susceptibility mapping in the brain. Journal of Magnetic Resonance Imaging, 42(1), 23-41. https://doi.org/10.1002/jmri.24768

Manley, G. T. M. D., MacDonald, C. L., Markowitz, A., Stephenson, D., Robbins, A., Gardner, R. C., ... Wang, K. K. W. 2017. The traumatic brain injury endpoints development (TED) initiative: Progress on a public-private regulatory collaboration to accelerate diagnosis and treatment of traumatic brain injury. Journal of Neurotrauma. https:// doi.org/10.1089/neu.2016.4729

Manley, G. T., Rosenthal, G., Lam, M., Morabito, D., Yan, D., Derugin, N., ... Panter, S. S. (2006). Controlled cortical impact in swine: Pathophysiology and biomechanics. Journal of Neurotrauma, 23(2), 128-139. https://doi.org/10.1089/neu.2006.23.128

Mannix, R., O'Brien, M. J., \& Meehan, W. P. 3rd. (2013). The epidemiology of outpatient visits for minor head injury: 2005 to 2009. Neurosurgery 73(1), 129-134; discussion 134.

McKee, A. C., \& Daneshvar, D. H. (2015). The neuropathology of traumatic brain injury. Handbook of Clinical Neurology, 127, 45-66.

Obenaus, A., \& Badaut, J. (2017). Noninvasive imaging techniques for brain edema: From basic science to the clinic. In J. Badaut, \& N Plesnila (Eds.), Brain edema (pp. 49-70). Oxford, UK: Academic Press.

Obenaus, A., Dilmac, N., Tone, B., Tian, H. R., Hartman, R., Digicaylioglu, M., ... Ashwal, S. (2011). Long-term magnetic resonance imaging of stem cells in neonatal ischemic injury. Annals of Neurology, 69(2), 282-291. https://doi.org/10.1002/ana.22168

Obenaus, A., Huang, L., Coats, J., Hartman, R., Badaut, J., \& Ashwal, S. (2012a). Animal models of mild pediatric TBI (2012). In M. W. Kirkwood, \& K. O. Yeates (Eds.), Mild Traumatic Brain Injury in Children and Adolescents: From Basic Science to Clinical Management (pp. 53-76). ISBN 978-1-4625-0513-5.

Obenaus, A., Huang, L., Coats, J. S., Hartman, R., Badaut, J., \& Ashwal, S. (2012b). Animal models. New York, NY: The Guilford Press.

Obenaus, A., Robbins, M., Blanco, G., Galloway, N. R., Snissarenko, E., Gillard, E., ... Curras-Collazo, M. (2007). Multi-modal magnetic resonance imaging alterations in two rat models of mild neurotrauma. Journal of Neurotrauma, 24(7), 1147-1160. https://doi.org/10.1089/neu.2006.0211

Oehmichen, M., Walter, T., Meissner, C., \& Friedrich, H. J. (2003). Time course of cortical hemorrhages after closed traumatic brain injury: Statistical analysis of posttraumatic histomorphological alterations. Journal of Neurotrauma, 20(1), 87-103. https://doi. org/10.1089/08977150360517218

Potts, M. B., Koh, S. E., Whetstone, W. D., Walker, B. A., Yoneyama, T., Claus, C. P., ... Noble-Haeusslein, L. J. (2006). Traumatic injury to the immature brain: Inflammation, oxidative injury, and iron-mediated damage as potential therapeutic targets. NeuroRx: The Journal of the American Society for Experimental NeuroTherapeutics, 3(2), 143-153. https://doi.org/10.1016/j.nurx.2006.01.006

Rodriguez-Grande, B., Obenaus, A., Ichkova, A., Aussudre, J., Bessy, T., Barse, E., ... Badaut, J. (2018). Gliovascular changes precede white matter damage and long-term disorders in juvenile mild closed head injury. Glia. https://doi.org/10.1002/glia.23336

Rowe, R. K., Harrison, J. L., Ellis, T. W., Adelson, P. D., \& Lifshitz, J. (2018). Midline (central) fluid percussion model of traumatic brain injury in pediatric and adolescent rats. Journal of Neurosurgery Pediatrics, 22(1), 22-30. https://doi.org/10.3171/2018.1.PEDS17449

Sandhir, R., Onyszchuk, G., \& Berman, N. E. (2008). Exacerbated glial response in the aged mouse hippocampus following controlled cortical impact injury. Experimental Neurology, 213(2), 372-380. https://doi. org/10.1016/j.expneurol.2008.06.013

Sarkar, K., Keachie, K., Nguyen, U., Muizelaar, J. P., Zwienenberg-Lee, M., \& Shahlaie, K. (2014). Computed tomography characteristics in pediatric versus adult traumatic brain injury. Journal of Neurosurgery Pediatrics, 13(3), 307-314. https://doi.org/10.3171/2013.12.PEDS13223

Schmued, L. C., \& Hopkins, K. J. (2000). Fluoro-Jade: Novel fluorochromes for detecting toxicant-inducedneuronal degeneration. Toxicologic Pathology, 28(1), 91-99. https://doi.org/10.1177/019262330002800111

Semple, B. D., Blomgren, K., Gimlin, K., Ferriero, D. M., \& NobleHaeusslein, L. J. (2013). Brain development in rodents and humans: Identifying benchmarks of maturation and vulnerability to injury across species. Progress in Neurobiology, 106-107, 1-16. https://doi. org/10.1016/j.pneurobio.2013.04.001 
Semple, B. D., Carlson, J., \& Noble-Haeusslein, L. J. (2016). Pediatric rodent models of traumatic brain injury. Methods in Molecular Biology, 1462, 325-343.

Siebold, L., Obenaus, A., \& Goyal, R. (2018). Criteria to define mild, moderate, and severe traumatic brain injury in the mouse controlled cortical impact model. Experimental Neurology, 310, 48-57. https://doi. org/10.1016/j.expneurol.2018.07.004

Thurman, D. J. (2016). The epidemiology of traumatic brain injury in children and youths: A review of research since 1990. Journal of Child Neurology, 31(1), 20-27. https://doi.org/10.1177/ 0883073814544363

Tong, K. A., Ashwal, S., Obenaus, A., Nickerson, J. P., Kido, D., \& Haacke, E. M. (2008). Susceptibility-weighted MR imaging: A review of clinical applications in children. AJNR American Journal of Neuroradiology, 29(1), 9-17. https://doi.org/10.3174/ajnr.A0786

Wendel, K. M., Lee, J. B., Affeldt, B. M., Hamer, M., Harahap-Carrillo, I. S., Pardo, A. C., \& Obenaus, A. (2018). Corpus callosum vasculature predicts white matter microstructure abnormalities after pediatric mild traumatic brain injury. Journal of Neurotrauma. https://doi. org/10.1089/neu.2018.5670

Yu, S., Kaneko, Y., Bae, E., Stahl, C. E., Wang, Y., van Loveren, H., ... Borlongan, C. V. (2009). Severity of controlled cortical impact traumatic brain injury in rats and mice dictates degree of behavioral deficits. Brain Research, 1287, 157-163. https://doi.org/10.1016/j.brainres.2009.06.067

Yue, J. K., Winkler, E. A., Burke, J. F., Chan, A. K., Dhall, S. S., Berger, M. S., ... Tarapore, P. E. (2016). Pediatric sports-related traumatic brain injury in United States trauma centers. Neurosurgical Focus, 40(4), E3.
Zhang, L., Hu, R., Li, M., Li, F., Meng, H., Zhu, G., ... Feng, H. (2013). Deferoxamine attenuates iron-induced long-term neurotoxicity in rats with traumatic brain injury. Neurological Sciences: Official Journal of the Italian Neurological Society and of the Italian Society of Clinical Neurophysiology, 34(5), 639-645. https://doi.org/10.1007/ s10072-012-1090-1

Zhao, S., Wang, X., Gao, X., \& Chen, J. (2018). Delayed and progressive damages to juvenile mice after moderate traumatic brain injury. Scientific Reports, 8(1), 7339. https://doi.org/10.1038/ s41598-018-25475-9

\section{SUPPORTING INFORMATION}

Additional supporting information may be found online in the Supporting Information section at the end of the article.

Transparent Science Questionnaire for Authors.

How to cite this article: Badaut J, Adami A, Huang L, Obenaus A. Noninvasive magnetic resonance imaging stratifies injury severity in a rodent model of male juvenile traumatic brain injury. J Neurosci Res. 2019;00:1-12. https:// doi.org/10.1002/jnr.24415 